\title{
Bubble Motion and its Surrounding Liquid Motion through the Collision of a pair of Bubbles"
}

\author{
Daiji SONE ${ }^{* *}$, Kazuya SAKAKIBARA ${ }^{* *}$, Masato YAMADA ${ }^{* *}$, \\ Toshiyuki SANADA $^{* *}$ and Takayuki SAITO** \\ **Department of Mechanical Engineering, Shizuoka University, \\ 3-5-1 Johoku, Hamamatsu, Shizuoka 432-8561, Japan \\ E-mail: ttsaito@ipc.shizuoka.ac.jp
}

\begin{abstract}
The interaction between bubble motion and its surrounding liquid motion through a collision of a pair of zigzagging bubbles in a rest water column was experimentally investigated. A pair of hypodermic needles and a bubble generator utilizing pressure oscillation were employed in order to exactly extract and highly reproduce the interaction between liquid-phase motion and bubble motion. The recursive cross-correlation PIV technique made it possible to obtain the accurate velocity field of surrounding liquid motion around a pair of bubbles. The vorticity field around a pair of bubbles was calculated from the results of velocity field. First, various kinds of interactions (e.g. bouncing and coalescence) were found out from the results of bubble motion (e.g. velocity) after a collision. We classified such interactions of a pair of bubbles using the dimensionless number $l / d$. Second, we investigated surrounding liquid motion about the two cases of interactions after the bouncing. One is that only horizontal velocity of bubble decreased after the collision, the other is that both horizontal and vertical velocity decreased. At the former case, the vortical region generated at the rear of a pair of bubbles. Therefore as the reason for the decrease of bubble velocity, the shedding area of hairpin-like vortex is restricted. At the latter case, bubble wake flows into the area between bubbles and vortical region doesn't generate under bubbles after the collision. Hence, bubble wake overtakes a pair of bubbles after the collision, then, bubble velocity decreased.
\end{abstract}

Key words: a pair of Bubbles, Collision, Interaction, Bouncing and Coalescence, Surrounding Liquid Motion, Vorticity

\section{Introduction}

Gas-Liquid bubbly flows are encountered in a lot of plants in a wide range of industrial fields, e.g. heat exchangers and chemical reactors. In addition, the bubbly flows are also applied to the field of environmental technology, such as GLAD system (e.g. Saito et al., $\left.1999^{(1)}, 2000^{(2)}, 2001^{(3)}\right)$. It is necessary for an optimal design and a control of those plants and systems to understand the structure and the mechanism of bubbly flows.

Bubble diameter and the number density of bubble are the dominant factors affecting specific features of a bubbly flow (e.g. Azbel, $1981^{(4)}$ ). In addition, the mass transfer coefficient for a single bubble depends on bubble diameter (Motarjemi et al., $1978^{(5)}$ ); the coefficient for $\mathrm{CO}_{2}$ takes the maximum when the equivalent diameter of bubble is about 2 
$\mathrm{mm}$. Furthermore, the values of mass transfer coefficient for oxygen adsorption in tap water are high against bubbles of diameter ranging from 2 to $5 \mathrm{~mm}$. Hence, those bubbles are important in industrial fields, especially in chemical field (e.g. bubble column). However, those bubbles rising in a rest liquid take on some different motions (e.g. deformation and trajectory) compared with small bubbles rising in a rest liquid; it is known that the small bubbles remain spherical, and their trajectories are rectilinear. Significant deformations occur in the case of diameters larger than $1 \mathrm{~mm}$; such bubbles take on a shape of a spheroid. Therefore, many studies regarding such bubbles as well as the small bubbles have been conducted; for example, studies on the zigzagging motion and the drift (the trajectory displacement) (e.g. Brücker, C., $1999^{(6)}$ and Ellingsen et al., 2001 ${ }^{(7)}$ ). Recently, the relation between zigzagging motion and interface deformation is investigated by measuring the curvature of left and right edge of a bubble (Miyamoto and Saito, $2005^{(8)}$ ). The studies on a single bubble rising in a rest liquid are essential, because this situation is elementary for bubbly flows. In addition, it is inevitable to investigate interactions (e.g. bubble-bubble interaction and bubble-wall interaction).

In the earlier studies of bubble-bubble interaction, Duineveld investigated the behaviour of bubbles which bounced or coalesced in super purified water. They suggested the border between bouncing and coalescence of bubble using Weber number (Duineveld, 1995 ${ }^{(9)}$ ). Sanada et al. investigated the interaction of a pair of bubbles which rise in a vertical line and side by side. They classified the interaction between bubbles with the use of Reynolds number (Sanada et al., 2006 $\left.{ }^{(10)}\right)$. As mentioned above, some researcher classified the interaction of two bubbles by using some dimensionless numbers. As the numerical analysis, the following model was suggested (Kirkpatrick et al., 1974 ${ }^{(11)}$, Chesters et al., $1982^{(12)}$ ); if the approach velocity of bubble is high, the pressure of liquid film becomes high and two bubbles bounce. On the other hand, if the approach velocity of bubble is low, two bubbles coalesce. Hence, the surrounding liquid of bubble plays the significant role during the collision of two bubbles. However, the experimental knowledge of surrounding liquid of bubble during the collision of two bubbles is still insufficient.

In the present study, bubble motion and its surrounding liquid motion through the collision of a pair of bubbles rising side by side was experimentally investigated. The PIV was employed in order to obtain the accurate velocity field around a pair of bubbles. The vorticity field was calculated from the PIV results. At first, we investigated kinds of interactions from the results of bubble motion (i.e. velocity of bubble). Then we classified the interactions (e.g. bouncing and coalescence) at each bubble using the dimensionless number $l / d$. Second, about the two types of interactions after a bouncing, we investigated the surrounding liquid motion during a bouncing. As the results, we found out the different liquid motion (e.g. velocity vector and vorticity) occurred between such two types of interactions. The effect of the surrounding liquid motion on bubble motion is discussed in view of the results of past studies about single bubble.

\section{Nomenclature}

$\begin{array}{llr}t: & \text { time } & {[\mathrm{msec}]} \\ u: & \text { velocity component of } x \text { direction } & {[\mathrm{mm} / \mathrm{s}]} \\ w: & \text { velocity component of } z \text { direction (vertical) } & {[\mathrm{mm} / \mathrm{s}]} \\ x, z: & \text { coordinate } & \\ s: & \text { distance of bubbles } & {[\mathrm{mm}]} \\ d: & \text { bubble diameter } & {[\mathrm{mm}]} \\ D_{\text {eq }}: & \text { equivalent bubble diameter } & {[\mathrm{mm}]} \\ L_{\text {needle }}: & \text { distance between needles } & {[\mathrm{mm}]}\end{array}$




\section{Experimental setup and analysis}

\subsection{Experimental setup}

Figure 1 shows a schematic diagram of experimental setup. An acrylic water vessel (a) $\left(150 \times 150 \times 300 \mathrm{~mm}^{3}\right)$ was filled with water to a depth of $270 \mathrm{~mm}$. The water is ion exchanged and degassed tap water. A pair of pure-air bubbles were launched simultaneously from a bubble launching device; here the device consists of audio speakers and a pair of hypodermic needles (b) (Inner diameter: $0.4 \mathrm{~mm}$ ) set on the bottom of the water vessel. The top of hypodermic needle is inclined and we faced the inclined surface of needles each other. A laser sensor (c) was placed as its beam intersected the zigzag trajectory of one of the pair bubbles to detect a bubble passing. The TTL signal from the laser sensor was inputted to a function generator (i). The digital recording of a high-speed video camera (Vision Research Corporation, Phantom V9.0) (d) was triggered by a functional pulse wave from the function generator.

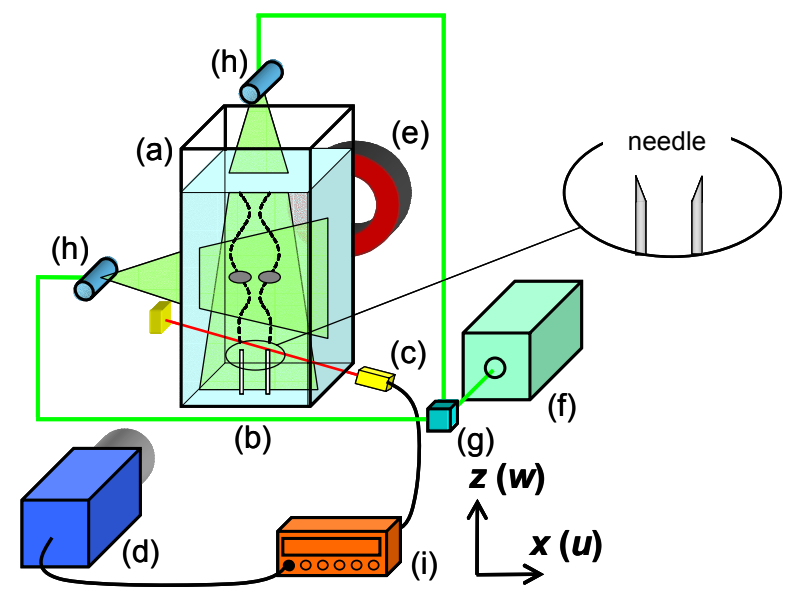

(a) Acrylic water vessel, (b) Hypodermic needle, (c) Laser sensor,

(d) High-speed video camera, (e) Continuous LED, (f) CW-YAG laser,

(g) Half mirror, (h) Rod lens, (i) Function generator

Fig. 1 Schematic of experimental setup.

The liquid phase motion was calculated as an ensemble average of sixteen dataset of PIV in order to obtain the reliable velocity field around bubbles. For this purpose, it is essential to reproduce and synchronize the formation of a pair of bubbles precisely. Hence, we adopted both hypodermic needles (i.e. control of initial bubble shape; Tomiyama et al., $2002^{(13)}$; Miyamoto and Saito, 2005 $5^{(14)}$ ) and audio speakers (i.e. control of pressure oscillation; Sanada et al., $\left.2006^{(10)}\right)$.

The hypodermic needles were able to make uniform bubbles in diameter, orientation, initial deformation and trajectory. The average equivalent diameter of bubble at the collision of a pair of bubbles is $2.9 \mathrm{~mm}$ in this study. The examined bubble was categorized into an oblate spheroid, and took zigzagging motion in a rest water column (Crift et al., $1978^{(15)}$ ). In this experiment, such bubble rose with two-dimensional zigzagging motion in the $x-z$ plane. During the zigzagging motion, the trajectory displacement called drift was observed. A pair of bubbles was collided on their paths by utilizing the drift phenomena.

The audio speakers were able to synchronize a bubble formation as well as a launch of bubbles by utilizing a well controlled pressure oscillation. In addition, the audio speakers were able to vary bubble diameter by transforming a pulse signal. Figure 2 shows a control unit for bubble formation and launch. Pure air was supplied from a cylinder (1). The pressure and air flow rate were controlled respectively via pressure gauge (2) and flow meter (3). They were kept constant all through every series of experimental run. A pulse signal of given amplitude and frequency was sent from a function generator (6) into an 
audio speaker (8) via power amplifier (7) (Kariyazaki et al., 1999 $\left.{ }^{(16)}, 2001^{(17)}, 2002^{(18)}\right)$. An additional pressure oscillation was exerted on air flow from the audio speaker. A pair of bubbles was formed and released from the hypodermic needles. The time intervals of launching a pair of bubbles were 5 seconds (Sanada et al., 2005 ${ }^{(19)}$ ). Such time is enough for dissipation of the liquid-phase motion induced by former bubbles. Hence, the effect of wake induced by former bubbles can be negligible after the interval time.

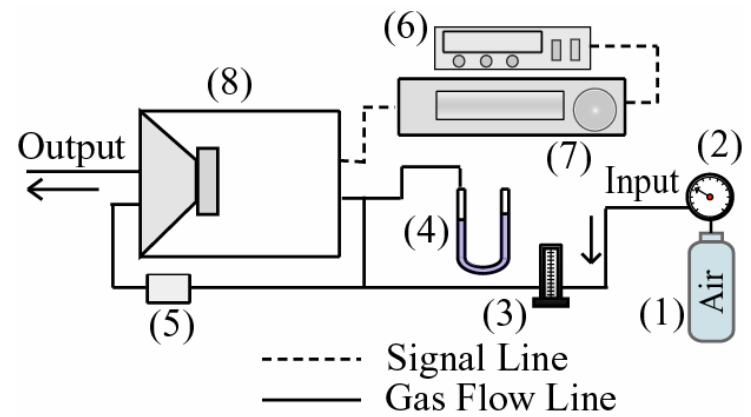

(1) Pure air tank, (2) Pressure gauge, (3) Flowmeter, (4) Manometer, (5) Check valve,

(6) Function generator, (7) Power amplifier, (8) Audio speaker

Fig. 2 Control unit for bubble generation.

\subsection{Analysis in experiments}

\subsubsection{Measurement of bubble diameter}

The average equivalent diameter of fifty sampled bubbles was $2.9 \mathrm{~mm}$. The bubbles were visualized, using a ring-shaped continuous red-LED array (e) (wavelength 660nm) and a high-speed video camera (d) (exposure time $200 \mu$ s, resolution $960 \times 960$ pixels, and spatial resolution $6.19 \mu \mathrm{m} / \mathrm{pixel}$ ). The bubbles were captured right after the launch. For a short time after the launch, bubble is almost oblate spheroid flattened at the $z$ axis like Fig. 3. In our earlier study (Miyamoto and Saito, $2005^{(8)}$ ), the equivalent bubble diameter derived from the way mentioned above was same as that derived from a direct sampling method. The error was about $1 \%$ of a equivalent bubble diameter.

Figure 3 shows a sample of bubble images for a measurement of bubble diameters from visualization. Using the LED as the back light, we were able to obtain images with the strong contrast between the bubble contour and the back ground. In addition, due to such strong contrast, this lighting method was very effective to quantitatively observe the bubble deformation.

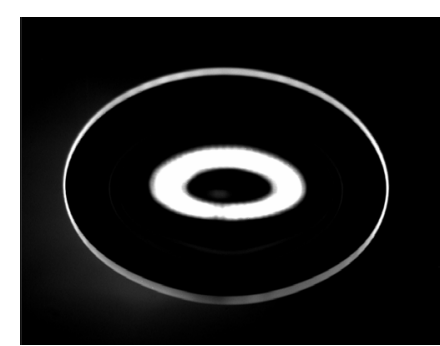

Fig. 3 Sample image of bubble using LED as the back light.

First, the centre-of-gravity positions and the contours of bubbles were obtained from a series of digital image processing (e.g. median filter, binarization, labeling, and so on). The threshold of a binarization was determined from the histogram of the gray-scale value of the original image. Second, the coordinates of contour were transformed from the two-dimensional rectangular coordinate system $(x, y)$ to the two-dimensional polar coordinate system $(r, \theta)$ by applying a centre-of-gravity position to a base point. The bubble shape was reconstructed by applying DFT (Discrete Fourier Transform) to the contour 
coordinate data. The bubble contour $r(\theta)$ was a periodic function of $\theta$ with a period of $2 \pi$, and expressed by the Fourier series given in Eq.(1).

$$
r(\theta)=A_{0}+\sum_{n=2}^{N} A_{n} \cos n \theta+\sum_{n=2}^{N} B_{n} \sin n \theta
$$

Duineveld reported that an accurate description of bubble shape could be obtained with a number of modes typically less than 8 (Duineveld, $1995^{(9)}$ ). Hence, $N=8$ was applied to Eq.(1).

From the results of Eq.(1), both major and minor axes were calculated. The bubble was considered to be categorized into an oblate spheroid flattened at the $z$ direction in the region in which the digital image of bubble was captured. Hence, the equivalent diameter $D_{e q}$ of bubble was calculated from the following equation;

$$
D_{e q}=\left(D_{\text {major }}^{2} \cdot D_{\text {minor }}\right)^{\frac{1}{3}}
$$

here, $D_{\text {major }}$ is the major axis and $D_{\text {minor }}$ is the minor axis.

\subsubsection{Measurement of bubble motion}

The motion of a pair of bubbles was visualized using a rectangular continuous red-LED array and a high-speed video camera (d) (exposure time $350 \mu \mathrm{s}$, frame rate $2000 \mathrm{fps}$, resolution $960 \times 432$ pixels, spatial resolution $0.17 \mathrm{~mm} /$ pixel).

The digital images were processed by almost the same way described in $\S 3.2 .1$. From such image processing, the trajectory of centre-of-gravity of each bubble was obtained. In addition, the horizontal and vertical velocity components of bubble motion were calculated from a change of the centre-of-gravity position every $0.5 \mathrm{~ms}$.

\subsubsection{Measurement of the surrounding liquid motion of a pair of bubbles}

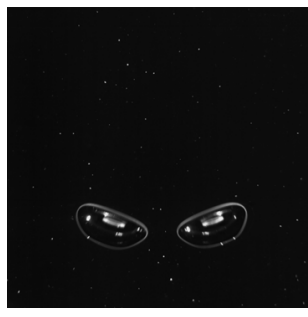

image-1 $(t)$

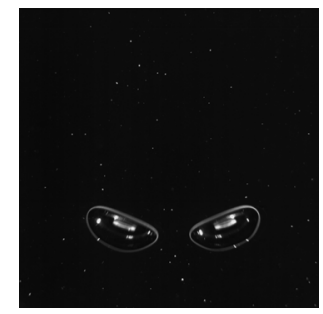

image-2 $(t+526 \mu \mathrm{s})$

(a) original images.

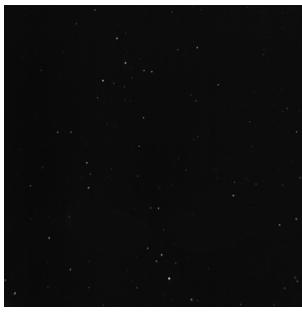

image-1 $(t)$

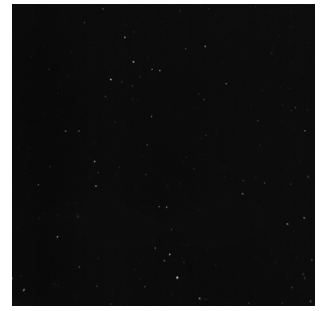

image-2 $(t+526 \mu \mathrm{s})$

(b) images after removing the bubbles.

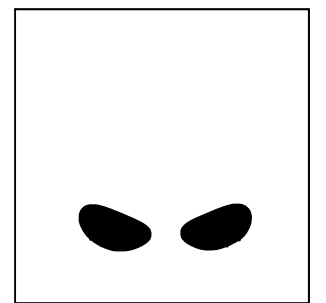

image-1 $(t)$

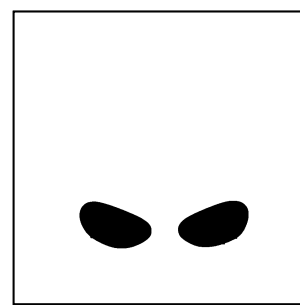

image-2 $(t+526 \mu \mathrm{s})$

(c) images of the bubbles

Fig. 4 Image pre-processing for PIV.

The liquid motion around a pair of bubbles through the collision was captured by a high-speed video camera (d) (exposure time $200 \mu \mathrm{s}$, frame rate $1960 \mathrm{fps}$, resolution $960 \times 960$ pixels, and spatial resolution $13.1 \mu \mathrm{m} / \mathrm{pixel})$.

As the PIV measurement, laser fluorescence particles (average diameter: $8 \mu \mathrm{m}$, excitation wavelength: $532 \mathrm{~nm}$, emission wavelength: $570 \mathrm{~nm}$ ) and CW-YAG laser (wavelength: $532 \mathrm{~nm}$ ) (f) were employed. In the experiment for bubble-bubble collision 
(Fig.1(A)) the laser beam was split into two directions by half mirror (g). Each laser beam became sheet-like illumination through rod lenses $(\mathrm{h})$, and illuminated a pair of bubbles from two sides; one is upper side and the other is left side. Hence, particles in the measurement area were illuminated uniformly.

Figure 4 shows the image pre-processing for PIV. The image-1 is the original image at certain time, and the image- 2 is the original image captured after $526 \mu \mathrm{s}$. First, the pair of bubbles (Fig.4(c)) was removed from the original images (Fig.4(a)) in order to extract only fluorescent particles (Fig.4(b)) for PIV process. Second, from the image of tracer particles, the velocity field of liquid around the pair of bubbles was calculated using PIV algorithm with a FFT-based recursive cross-correlation method; the interrogation region was downsized from $64 \times 64$ pixels to $32 \times 32$ pixels. The velocity field of the surrounding liquid at each stage during a bubble-bubble collision was obtained as the average of sixteen times data of corresponding PIV measurement.

A recursive cross-correlation method (Hart D. P., $1999^{(20)}, 2000^{(21)}$ ) increased the spatial resolution and also prevented the generation of incorrect vectors. In the PIV measurement, the spatial resolution is limited by the number of tracer particles in interrogation region. If the number of tracer particles in the interrogation region is not enough, the error vector increases. However, this method achieves the reliable velocity measurement even if the number of tracer particles in the interrogation region is small; not only such advantages, but also this method minimizes the effect of tracer particles on bubble motion as well. A small amount of contamination of water may seriously affect the behavior of a bubble surface; usually surface-contaminated bubble behaves like a rigid body. Hence, it is better for the accurate measurement of surrounding liquid motion of a pair of bubbles to reduce the number of tracer particles in the interrogation region as long as possible. In our earlier study, we have applied the FFT-based recursive cross-correlation method to the measurement of liquid motion around a single rising bubble (e.g. Sakakibara and Saito. $2006^{(22)}, 2007^{(23)}$; Miyamoto and Saito, $\left.2005^{(24)}\right)$.

\section{Results and Discussion}

\subsection{Interaction of a pair of bubbles}

Samples of bubble motions of a pair of zigzagging bubbles are shown in Fig.5. The trajectory and the velocity of bubble at each case of Fig.5 are shown in Fig.6. The vertical axis in Fig. 6 shows an elapsed time after a bubble detaches from a hypodermic needle. $A$ in Fig. 5 is the first approaching point of a pair of bubbles and $B$ is the second approaching point. In order to categorize the interactions of a pair of bubbles, we use the dimensionless number $l / d$ ( $l$ means the distance between centre-of-gravity of each bubble, $d$ means the equivalent bubble diameter $D_{e q}$ ).

At first, we discuss the interaction when a pair of bubbles doesn't collide. At the case (a), bubbles rise with zigzagging motion shown in Fig.5(a) and the trajectory and the velocity of a pair of bubbles are same as those of single bubble after $A$ and $B$ in Fig.6(a). Hence, in this case, there is no interaction of a pair of bubbles. Then, at the case (b), bubbles approach closer by making needles approach closer than case (a). As the results, the vertical velocity of a pair of bubbles is almost same as that of single bubble after $A$ and $B$ shown in Fig.6(b). On the other hand, the horizontal velocity of a pair of bubbles is not same as that of single bubble after $A$ and $B$; comparing the oscillation of horizontal velocity of a pair of bubbles with that of single bubble, the oscillation of a pair of bubbles seems like the phase shifting of that of a single bubble. Hence, in this case, there is the interaction of a pair of bubbles even if a pair of bubbles doesn't collide. We classify whether bubbles interact or not interact when bubble don't collide using the dimensionless number $l / d$; this is discussed at the last part of this session. 


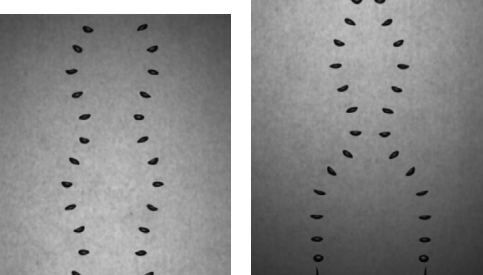

(b) $\mathrm{L}_{\text {needle }}=34.3$

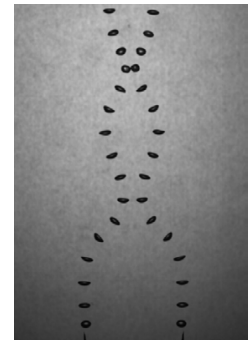

(c) $\mathrm{L}_{\text {needle }}=31.2$

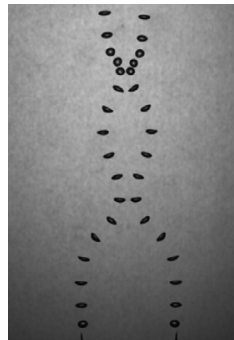

(d) $\mathrm{L}_{\text {needle }}=30.2$

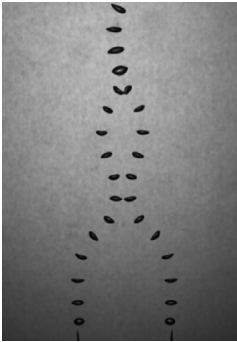

(e) $\mathrm{L}_{\text {needle }}=29.7$

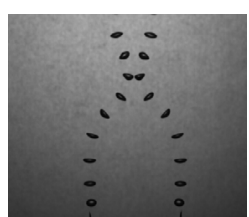

(f) $\mathrm{L}_{\text {needle }}=28.2$

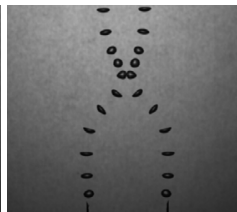

(g) $\mathrm{L}_{\text {needle }}=25.7$

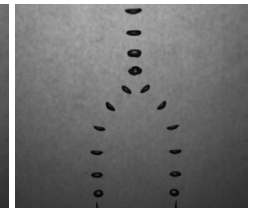

(h) $\mathrm{L}_{\text {needle }}=\mathbf{2 5 . 2}$

Fig. 5 Superimposed images of bubble every $25 \mathrm{~ms}$.

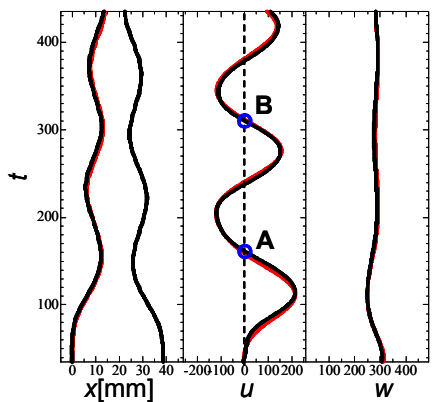

(a) $\mathrm{l} / \mathrm{d}=6.9$ at $(\mathrm{A}), 6.2$ at $(\mathrm{B})$

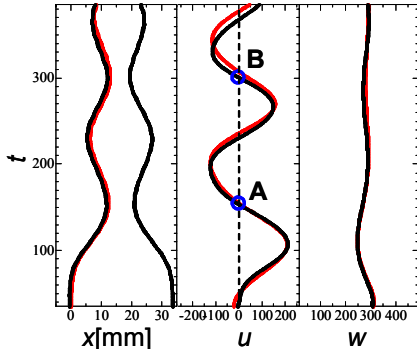

(b) $l / d=3.1$ at $(A), 2.5$ at (B)

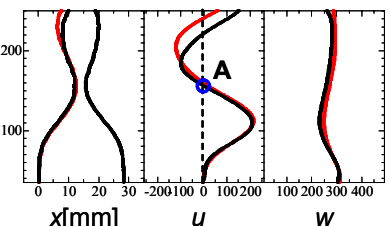

(f) $l / d=1.5$ at $(A)$

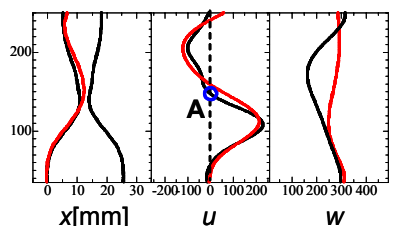

(g) $l / d=1.5$ at $(\mathrm{A})$

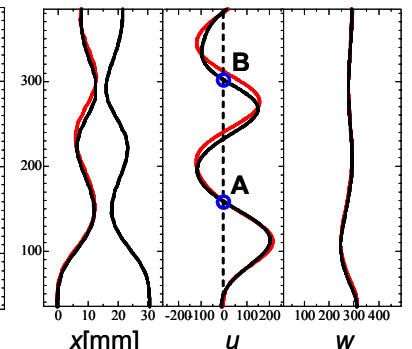

(c) $l / d=2.0$ at $(A), 1.5$ at $(B)$

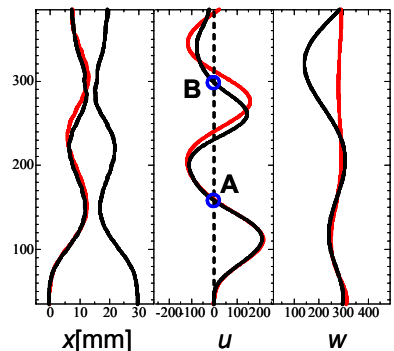

(d) $l / d=1.7$ at $(A), 1.5$ at $(B)$

A pair of bubbles( left bubble )

Single bubble

Fig. 6 Bubble trajectory and velocity profiles.

Second, we discuss the interaction when a pair of bubbles collides. After a collision, bouncing and coalescence are observed. At the case (c), after $A$ in Fig.6(c), the same kind of phenomena at the case (b) are observed. Then, bubbles collide at $B$; right before the inverse point of zigzagging motion shown in Fig.5(c). After the collision, bubbles bounce and only the horizontal velocity of bubble decrease shown in Fig.6(c); on the other hand, the vertical velocity of bubble doesn't decrease. At the case (d), after $A$ in Fig.6(d), the same kind of phenomena at the case (b) are observed. Then, after the collision at $B$, bubbles bounce and both horizontal and vertical velocity of bubble decrease shown in Fig.6(d). At the case (e), after the collision at $B$, a pair of bubbles coalesce shown in Fig.5(e). At the case (f), after the collision at $A$, bubbles bounce and only the horizontal velocity of bubble decrease shown in Fig.6(f); this phenomenon is same as that after the collision at the case (c). At the case (g), after the collision at $A$, bubbles bounce and both horizontal and vertical velocity of bubble decrease in Fig.6(g); this phenomenon is same as that after the collision at the case (d). At the case (h), after the collision at $A$, a pair of bubbles coalesce in Fig.5(h); this phenomenon 
is same as that after the collision at the case (e). Therefore, comparing the velocity fluctuation of bubble at the case (c), (d), (e) with that at (f), (g), (h), same kind of phenomena occurred after the collision at $A$ and $B$; as making bubbles approach closer, at first, only horizontal velocity decrease after the collision, then, both horizontal and vertical velocity decrease after the collision, finally, a pair of bubbles coalesce.

As we mentioned before, from the results of bubble motion, we found out there are several kinds of interactions of a pair of bubbles after the collision. Then, we categorized these interactions like Fig. 7 using the dimensionless number $l / d$. From Fig.7, if the distance between bubbles is more than three times as large as equivalent bubble diameter, bubbles don't collide and there is no interaction of a pair of bubbles. On the other hand, if the $l / d$ ranges from 1.5 to 3.1 , even if bubbles don't collide but the interaction which is seen at the case (b) occurred; the phase shifting of the oscillation of bubble horizontal velocity occurred. In addition, if the $l / d$ is less than 1.5 , bubbles collide and the interaction which seen at the case (c), (d), (e), (f), (g), and (h) occurred.

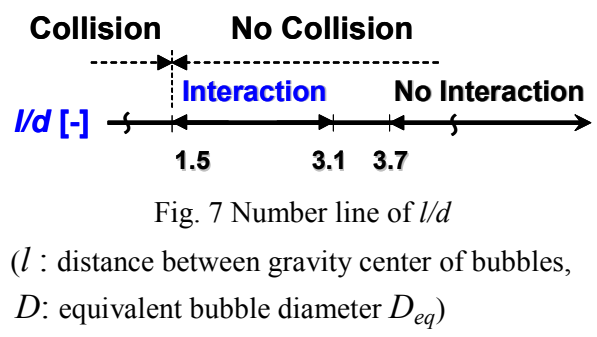

\subsection{Liquid motions around a pair of bubbles}

In this section, we discuss the difference of surrounding liquid motion of bubbles during the collision about two cases of interaction. The first one, interaction (1), is the interaction at the case (c) and (f); only horizontal velocity of bubble decreases after the collision. The second one, interaction (2), is the interaction at the case (d) and (g); both horizontal and vertical velocity decrease after the collision.

Fig. 8 shows the sketch of wake under a zigzagging bubble. The wake in Fig. 8 shows the hairpin-like vortex structures which is made under a zigzagging bubble. This sketch is drawn in view of past studies about a single bubble with zigzagging motion such as Brüker's study $^{(6)}$. Fig.9 is the images of our PIV results (velocity vector and vorticity) of surrounding liquid motion at the interaction (1). (b), (c), and (d) are the images past $t$ millisecond from (a). The black part of each image shows a pair of bubbles; the bubble images are pasted on the PIV results.

About the interaction (1), the velocity vector and the vorticity of surrounding liquid in Fig.9(a) is almost same as that of a single rising bubble (Sakakibara and Saito. $2007^{(23)}$ ); the measurement plane is the $x-z$ plane in which a pair of bubbles rose with zigzagging motion shown in Fig.8. Then, after the collision in Fig.9(b), bubbles deform rapidly and each bubble wake shown in Fig. 8 collides and interacts each other. In Fig.9(c), the vortical region generates at the rear of bubbles as the results of the interaction between each bubble wake. That vortical region is so strong that that region exists after a pair of bubbles gets away from each other. Considering the reason for the decrease of only horizontal velocity after the collision from the PIV results mentioned above, the shedding area of hairpin-like vortex shown in Fig. 8 is considered to be restricted. In the past studies, Brüker reported that a zigzag motion is coupled to a regular generation and the shedding of hairpin-like vortex structures. In addition, during the zigzag motion, only horizontal velocity of bubble changes; vertical velocity of bubble is almost same. Hence, that regular generation and the shedding of hairpin-like vortex structures associate only horizontal velocity. Then, after the collision, the shedding area of hairpin-like vortex is restricted. That is why, only horizontal velocity of bubble decreases after the collision. 

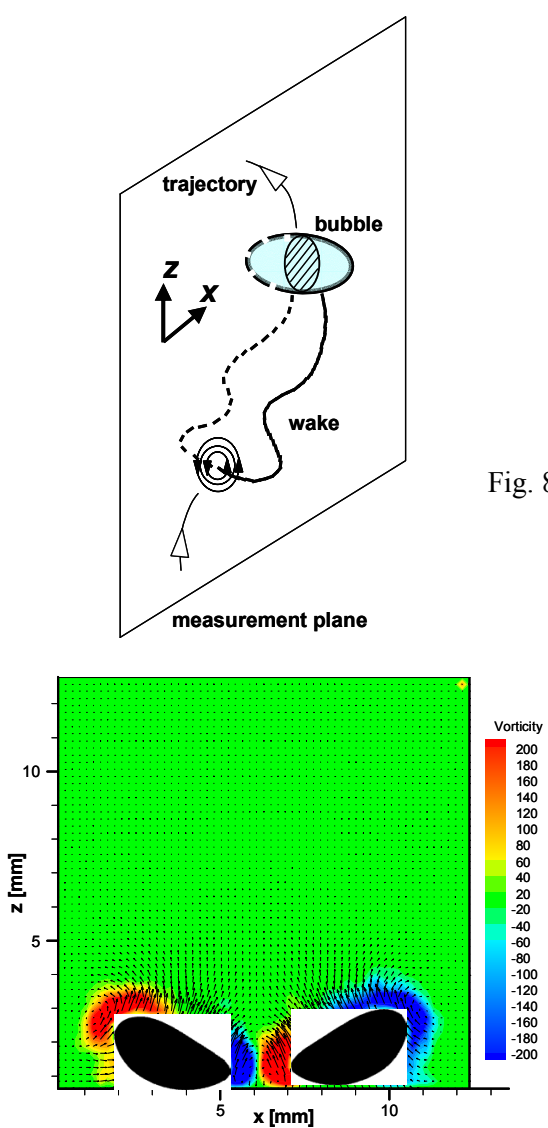

(a) Before collision $(t=0)$

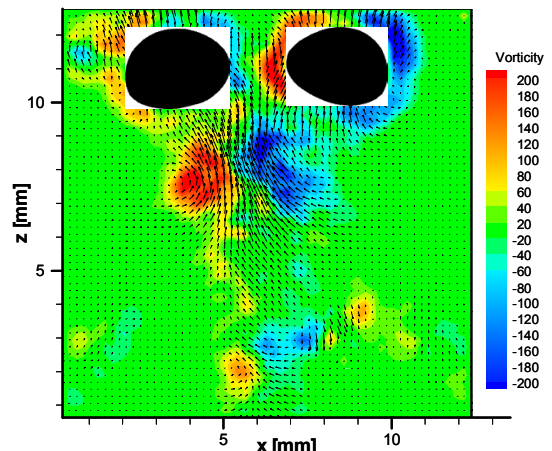

(c) After collision ( $t=36.8$ )
Fig. 8 Sketch of wake under a zigzagging bubble.

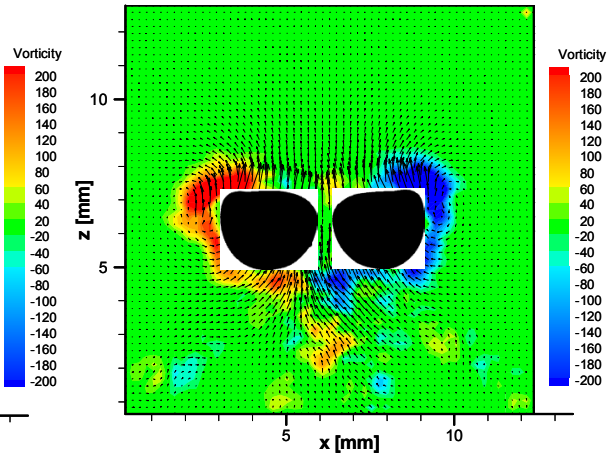

(b) After collision ( $t=17.9)$

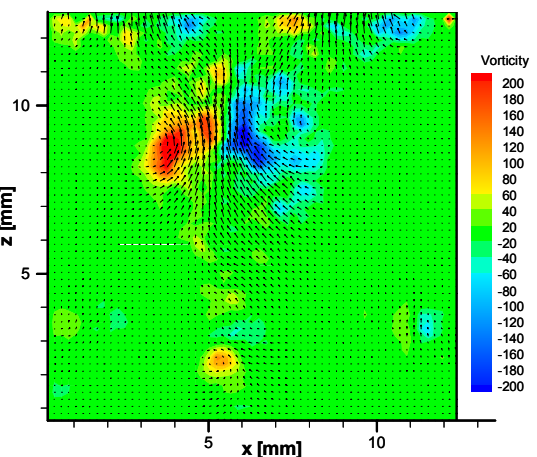

(d) After collision ( $t=46.8$ )

Fig. 9 Velocity vector and vorticity profiles at interaction (1).

Fig. 10 and 11 are images of our PIV results; Fig.10 shows the velocity vector and the vorticity and Fig.11 shows the vertical velocity and the velocity vector of surrounding liquid motion at the interaction (2). (b), (c), and (d) are the images past $t$ millisecond from (a).

About the interaction (2), the velocity vector and vorticity of surrounding liquid in Fig.10(a) is almost same as that of single rising bubble (Sakakibara and Saito. $2007^{(23)}$ ). Then, after the collision in Fig.10(b), bubbles deform rapidly and each bubble wake collides and interacts each other. The small vortical region generates at the rear of a pair of bubbles. This vortical region is similar to that in Fig.9(b) at the interaction (1). However, in Fig.10(c), that vortical region doesn't exist at the rear of bubbles. Comparing Fig.9(c) with Fig.10(c), the vortical region under bubbles obviously doesn't generate in Fig.10(c). This fact shows bubble wake may flow to somewhere else after the collision at the interaction (2). In Fig.9(d), large vortical region generates again at the rear of each bubble. 


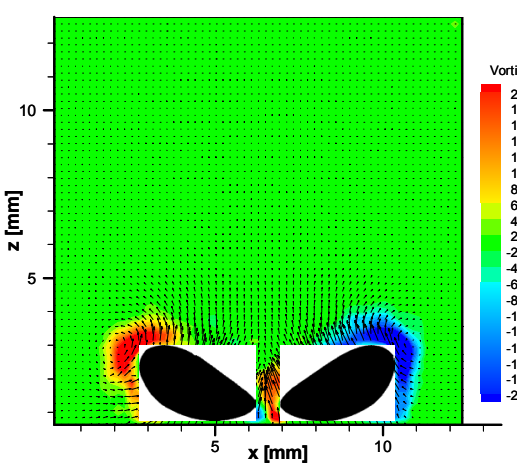

(a) Before collision $(t=0)$

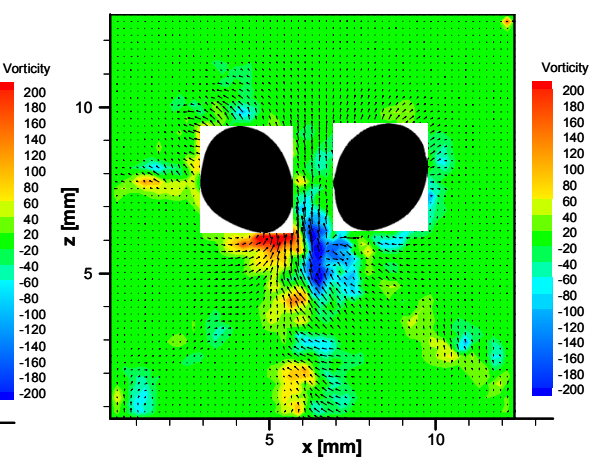

(b) After collision $(t=29.5)$

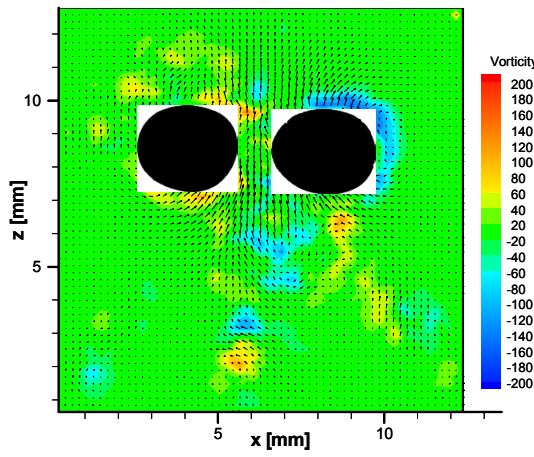

(c) After collision $(t=41.6)$

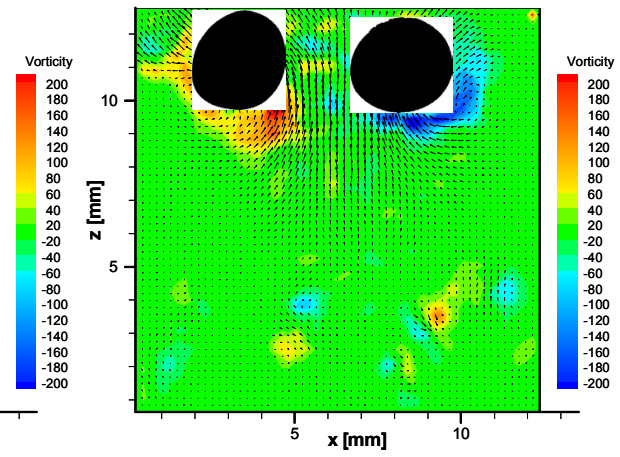

(d) After collision $(t=56.4)$

Fig. 10 Velocity vector and vorticity profiles at interaction (2).

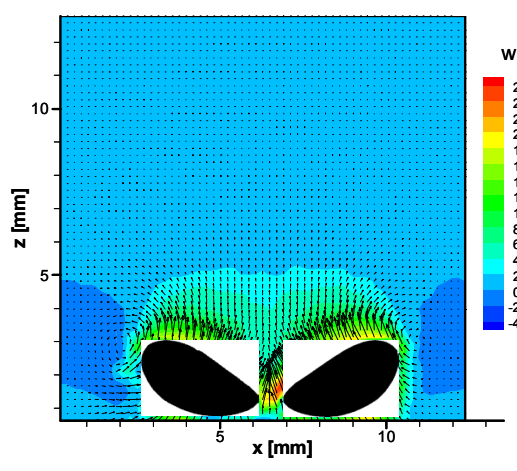

(a) Before collision $(t=0)$

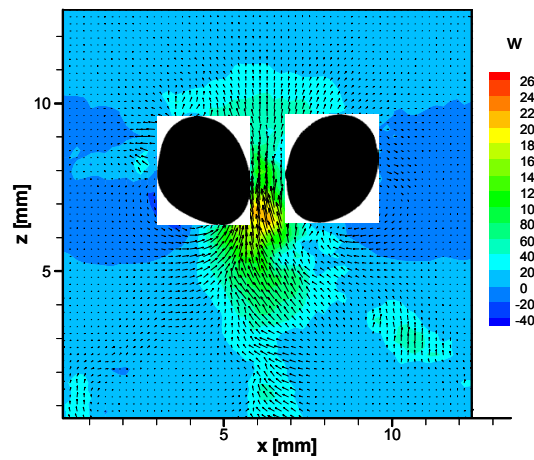

(c) After collision ( $t=29.9$ )

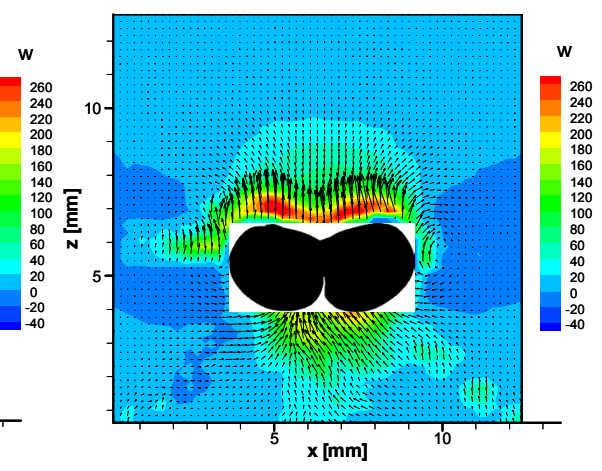

(b) After collision ( $t=15.7)$

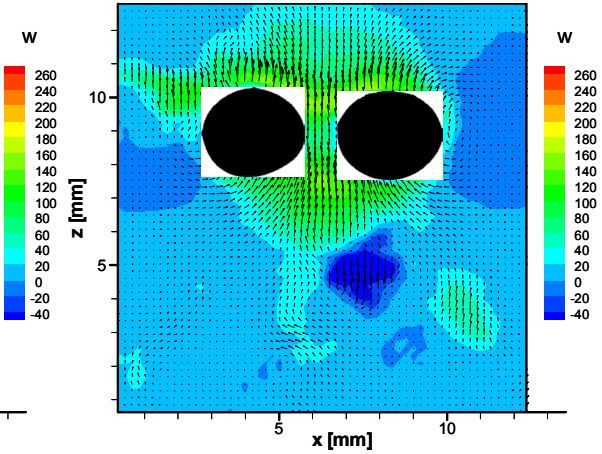

(d) After collision ( $t=43.6)$

Fig. 11 Vertical velocity and velocity vector profiles at interaction (2).

Then, we focus on the vertical velocity of surrounding liquid of bubbles before and after the collision of a pair of bubbles. Fig.11(a) shows that the vertical velocity of liquid on the upper side of each bubble is high before the collision; when a bubble rises by its buoyant force, the bubble pushes up upper liquid and the vertical velocity of liquid on the 
upper side of each bubble is close to the vertical velocity of each bubble. In addition, the same phenomenon is shown in Fig.11(b); this shows that the vertical velocity of each bubble doesn't decrease right after the collision. However, in Fig.11(c), the vertical velocity of liquid on the upper side of each bubble is low. On the other hand, the vertical velocity of liquid between a pair of bubbles is high. In fact, each bubble wake flows into the area between bubbles after the collision; the low vertical velocity of liquid on the upper side of each bubble shows that the vertical velocity of each bubble is low. In Fig.11(d), the vertical velocity of liquid on the upper side of each bubble becomes higher than that in Fig.11(c). This shows that the vertical velocity of each bubble becomes higher than that in Fig.11(c). Therefore, the fact which is made out from the above results is that each bubble wake flows into the area between bubbles after the collision.

From the PIV results in Fig.10 and 11 (vertical velocity, velocity vector, and vorticity) before and after the collision at the interaction (2), the vortical region shown in Fig.10(d) is considered to be generated mainly due to the liquid which flows into the area between bubbles to upper side of bubbles after the collision. Considering the reason for the decrease of both horizontal and vertical velocity of bubble after the collision from the PIV results mentioned above, after the collision, each bubble wake overtakes a pair of bubbles. Then, that liquid flow decreases the velocity of bubble.

\section{Conclusion}

We investigate the interaction between a pair of zigzagging bubble and classify such interactions using the dimensionless number $l / d$. Even if bubbles don't collide, we found out that the interaction occurred when $l / d$ ranges from 1.5 to 3.1. After the collision, when $l / d$ is 1.5 , bouncing and coalescence are observed. Then, after the bouncing, two types of unique interaction (i.e. bubble motion) occurred. The first one, interaction (1), is the interaction that only horizontal velocity of bubble decreases after the bouncing. The second one, interaction (2), is that both horizontal and vertical velocity decrease after the collision.

The PIV measurement is applied to investigate the effects of surrounding liquid motion on the bubble motion at interaction (1) and (2). As the results about interaction (1), the vortical region generate at the rear of a pair of bubbles. From this result and past studies, as the reason for the decrease of only horizontal velocity of bubble after the bouncing, the shedding area of hairpin-like vortex is considered to be restricted. As the results about interaction (2), the vortical region like that of interaction (1) doesn't generate under bubbles. In addition, from the PIV results, the liquid flows into the area between bubbles to upper side after the collision. Hence, from these two results, after the bouncing, each bubble wake overtakes a pair of bubble and then bubble velocity decrease.

\section{Acknowledgements}

The present study was promoted and financially supported by Category "A" of the Grants-in-Aid for Scientific Research, Japan Society for the Promotion of Science. We thank JSPS for its help in the financial support.

\section{References}

(1) Saito, T. Kajishima, T. Tsuchiya, K. and Kosugi, S., Mass transfer and structure of bubbly flows in a system of $\mathrm{CO} 2$ disposal into the ocean by gas-lift column, Chem. Eng. Sci., 54 (1999), pp.4945-4951.

(2) Saito, T. Kajishima, T. and Nagaosa, R., CO2 Sequestration at sea by gas-lift system of shallow injection and deep releasing, Environmental Sci. \& Tech., 34 (2000), pp.4140-4145.

(3) Saito, T. Kosugi, S. Kajishima, T. and Tsuchiya, K., Characteristics and performance of a deep ocean disposal system for low-purity CO2 gas via a gas lift effect, Energy \& Fuels, 
15 (2001), pp.285-292.

(4) Azbel, D., "Two-phase flows in chemical engineering”, Cambridge. (1981)

(5) Motarjemi, M. ans Jameson, G. J., Mass Transfer from Very Small., Bubbles-The Optimum Bubble Size for Aeration, Chem, Eng. Sci., Vol. 33 (1978), pp.1415-1423.

(6) Brüker, C., Structure and dynamics of the wake of bubbles and its relevance for bubble interaction., Physics of Fluids, 11 (1999), 781-1796.

(7) Ellingsen, K. and Risso, F., On the rise of an ellipsoidal bubble in water: oscillatory paths and liquid-induced velocity., J. Fluid Mech. Vol. 440 (2001), pp. 235-268.

(8) Miyamoto, Y. and Saito, T., Relationship between Interface Motion of an Isolated Bubble and its Zigzagging Motion of the Center of Gravity., J. Jpn. Soc. Mech. Eng., B71-705 (2005),1307-1313. (Japanese)

(9) Duineveld, P. C., The rise velocity and shape of bubbles in pure water at high Reynolds number., J. Fluid Mech., 292, 325-332. (1995)

(10) Sanada, T. Watanabe, M. and Fukano, T., Interaction and coalescence of bubbles in stagnant liquid., Multiphase Sci. Tech., 18 (2006), 155-174.

(11) Kirkpatrick, R. D., Lockett, M., J., "The influence of approach velocity on bubble coalescence”, Chem. Eng. Sci., 29 (1974), 2363-2373.

(12) Chesters, A. K., Hofman, G., "Bubble coalescence in pure liquid", Appl. Sci. Res., 38 (1982), 353-361.

(13) Tomiyama, A., Celata, G. P., Hosokawa, S., Yoshida, S., "Terminal velocity of single bubbles in surface tension force dominant regime”, Int. J. Multiphase Flow, 28 (2002), 1497-1519.

(14) Miyamoto, Y. and Saito, T., Experiments on Visualizing the pseudo-3D Structure of Bubble Shape and its Surrounding Liquid Motion., IASME Transactions, Vol. 2, Issue 9 (2005), pp. 1606-1611.

(15) Clift, R., Grace, J. R. and Weber, M. E., Bubbles, Drops, and Particles., Academic Press. (1978)

(16) Kariyazaki, A., Ousaka, A., and Kagawa,M., Effect of pressure oscillation on the bubble generation from a nozzle., Proceedings of the 3rd ASME/JSME Joint Fluids Eng. Conf. (1999), FEDSM 99-7846

(17) Kariyazaki, A., Ousaka, A., Control of bubble size and detaching frequency from a submerged nozzle by means of pressure oscillation induced in the gas flow., 6th World Congress of Chemical Engineering Melbourne, Australia 23-27. (2001)

(18) Kariyasaki, A., Ousaka, A., Control of bubble formation (effect of additional oscillation to gas velocity on bubble formation)., J. Jpn. Soc. Mech. Eng., B 68-674 (2002), 2712-2718. (Japanese)

(19) Sanada, T. Watanabe, M. Fukano, T. and Kariyasaki, A., Behavior of a single coherent gas bubble chain and surrounding liquid jet flow structure., Chemical Engineering Science, 60 (2005), 4886-4900.

(20) Hart, D. P., PIV Error Correction, Exp. Fluids, Vol. 29 (2000) pp. 13-22.

(21) Hart, D. P., Super-resolution PIV Processing by Recursive Correlation, J. Visualization, Vol. 2 (1999), pp. 187-194.

(22) Sakakibara, K. Yamada, M. , Miyamoto, Y. and Saito, T., Measurement of the surrounding liquid motion of a single rising bubble using a dual-camera PIV system., 5th ISMTMF Vol. 2, 1071-1076. (2006)

(23) Sakakibara, K. Yamada, M., Miyamoto, Y. and Saito, T. (2007), Measurement of the surrounding liquid motion of a single rising bubble using a dual-camera PIV system., Flow Measurement and Instrumentation, in press.

(24) Miyamoto, Y. and Saito, T., Bubble Deformation and Structure of its Surrounding Liquid Motion Measured via PIV-LIF., Proceedings of the 4th World Congress on Industrial Process Tomography (2005), pp.965 - 970. 